\title{
Research on Design of Plate-type Electromagnetic Coupler in Underwater Inductive Power Transmission
}

\author{
Li-yan $\mathrm{Qu}^{1}$ (Author), Dao-he Sun ${ }^{1, a}$ \\ ${ }^{1}$ Zhonghuan Information College Tianjin University of Technology
}

\begin{abstract}
Magnetic coupler has a good application in the field of underwater sensor. Magnetic coupler at work, interference by underwater complex situation, stability and efficiency of charging device of the gap is larger fluctuations. The traditional electromagnetic coupling is charging for the stability of the clearance to demand higher. Charging for underwater, as a result of the existence of ocean currents, electromagnetic coupling clearance may not remain very stable. When there is deviation gap, a larger electromagnetic coupling performance deviation. On this particular problem, it puts forward the design method of a new type of plate type electromagnetic coupling. First of all, the leakage inductance of the finite element method to calculate system and excitation inductance, establish electromagnetic coupler with compensation capacitor equivalent circuit, and the primary circuit and secondary circuit was designed. On the basis, the voltage gain and efficiency of the system are carrying on the theoretical derivation and calculation. The simulation experimental results show that the magnetic coupler has a stable voltage gain and charging efficiency, when the partial core within $10 \mathrm{~mm}$, voltage gain remains steady at $5.8 \%$, efficiency remain at around $90 \%$.
\end{abstract}

\section{Introduction}

As human demand for marine resources, more and more mechanical and electrical equipment will be used in underwater ocean probing. Because of the vastness of the ocean, the traditional mechanical and electrical equipment is limited in the use of distance, and cannot carry out the large-scale detection. Therefore, when the energy is exhausted, underwater equipment needed energy supplies. There are two main methods of energy supply. One is to be charged by the shore or underwater equipment deck, the other one is underwater wet pluggable charging by a cable system. The degree of automation about the two charging methods are low, and the wet plug insertion force required is large, which lead to serious wear and tear, and make a limit number of charge cycles. So the researchers tried to introduce the inductive power transfer device in the underwater to improve the reliability of the system. In foreign countries, the efficiency of $200 \mathrm{~W}$ underwater CLPT system researched and developed by Feezor and Sorrel can reach $79 \%{ }^{[1,2]}$; Tohoku University and NEC jointly developed 500W underwater CLPT system and the efficiency is above $90 \%$, the gap reached $2 \mathrm{~mm}{ }^{[3,4]}$; McGinni design 250W induction charging system for sectional observations of ocean mooring, which efficiency is more than $70 \%{ }^{[5]}$; The department of Electronic and Computer Network in Japan Sojo University and the Marine Engineering and Earth
Sciences institute of Japan and other institutions jointly develop a similar mooring charging system ${ }^{[6]}$; German MESA company has developed INPUD CAN for using in shallow water and INPUD DON system for using in deep water. The transmission power of this system is $100 \mathrm{~W}$, the efficiency reached $90 \%$. CLPT underwater research technology in China is still in its infancy. Tianjin University has carried out relevant research about ocean buoys underwater non-contact power supply ${ }^{[7]}$, Zhejiang University has carried out relevant research in the field of deep non-contact power transmission coupling ${ }^{[8] \text {, }}$ and made some progress. At present, the subsea equipment system developed successfully by China has no case of non-contact power supply technology ${ }^{[9]}$.

Considering the discovery of existing relevant literature comprehensively, charging equipment in underwater require the higher lever of the stability about the time of the charging gap. Electromagnetic coupling is in the work, because of underwater complex situations interference, stability and efficiency of the charging device's gap has a large fluctuation of the charging device. The stability of the traditional electromagnetic coupling require the higher charging gap .During underwater charging, due to the presence of ocean currents, the electromagnetic coupling is impossible to keep stable . When the gap deviation, a larger electromagnetic coupling will perform deviations.

${ }^{\mathrm{a} C}$ Corresponding author:sundaoheaixu@126.com. 
This paper presents a new flat electromagnetic coupling design. Firstly, calculate the leakage inductance and magnetizing inductance system by the finite element method to establish the electromagnetic coupling equivalent circuit with a compensation capacitor, and design the primary and secondary circuits. On this basis, the voltage gain and efficiency of the system are deduced theoretically and calculated.

\section{Principle of electromagnetic coupling design}

Design of the electromagnetic coupling is shown in Figure 1. The electromagnetic coupling eccentricity in the case of radial and axial displacement can maintain the stable magnetizing inductance and leakage inductance. Therefore, when the disturbance currents, it can keep stable charging performance.

Because of the core gap, a divergent magnetic field will induce eddy currents in surrounding metal housing. Eddy currents can play a role of an electromagnetic shielding that reduces electromagnetic coupling to external radiation. However, due to the larger shell thickness, the energy caused by eddy current losses. Thus, between the core and the shell, it needs to add additional shield to reduce the effects of electromagnetic fields and decrease shield energy loss. In addition, in order to protect the coil and reduce eddy current losses of the coil window, the installation of protective rubber is

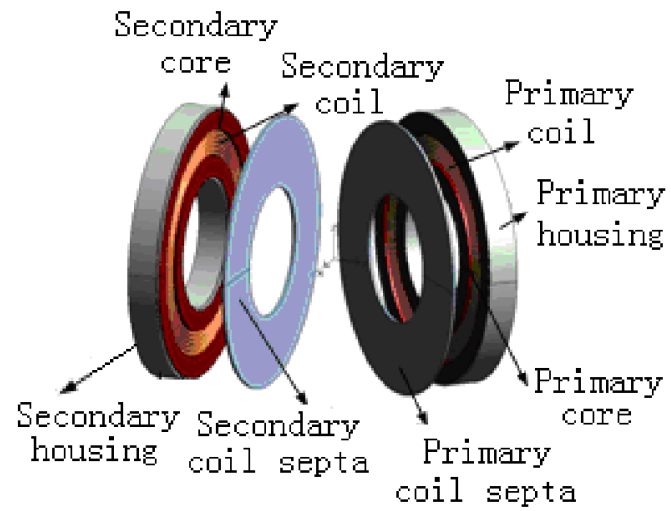

Figure 1. A schematic diagram of the electromagnetic coupling flatbed

septum in the coil window. Inductive power transfers into the equivalent circuit analysis and finite element method. The speed of the equivalent circuit method is fast, but the deviation of parameter calculation is large; finite element method is accuracy, but too time-consuming. In this paper, at first, the equivalent circuit method and finite element method are used comprehensively to analyze system performance. Leakage and magnetizing inductance system is calculated by finite element method. And then an equivalent circuit of the system is established, thereby, computing the gain and the transmission efficiency of the system voltage.

Inductive power transfer system is separate structure, low coupling coefficient, low power factor, and the system's transmission capacity is weak. Therefore, the compensative technology is required, which make the system to achieve maximum power factor to achieve energy efficient transmission by the resonant inductance compensation capacitor system. Underwater equipment inductive power transfer diagram is shown in Figure 2, "T"-type equivalent circuit with "ss" compensation is shown in Figure 3.

After the establishment of the equivalent circuit, the equivalent circuit parameters is required to calculated by

Figure 2. Schematic inductive power transfer

the various methods to further analyze system performance.

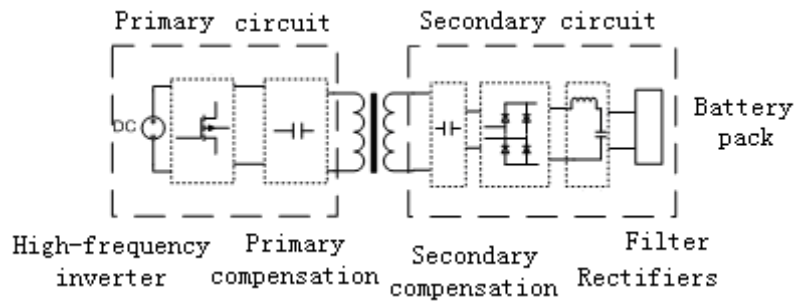

Figure 2 "SS" type compensator electromagnetic coupling "T"-type equivalent circuit

Calculate $R_{f}$ and $R_{s}$ firstly, switch circuit and the resonance circuit can improve the transmission efficiency of the system, but because of containing the non-sinusoidal current waveform component, which results in additional AC loss. In addition, since the effective utilization area of the skin effect and proximity effect, the wire is reduced. The effective impedance of the primary side and the secondary side can be expressed as:

$$
\begin{aligned}
R & =R_{d c}+\frac{\psi}{3} \Delta^{4} R_{d c}\left(\frac{I^{\prime}}{\omega \cdot I}\right) \\
\psi & =\frac{5 p^{2}-1}{15} \\
\Delta & =\frac{d_{\text {eff }}}{\delta_{0}} \\
\delta_{0} & =\sqrt{\frac{1}{\pi f_{s w} \mu_{0} \delta}} \\
R_{d c} & =4 \rho \frac{N \cdot M L T}{\pi \cdot S t r \cdot d_{c}^{2}}
\end{aligned}
$$

Among them $\omega=2 \pi f$

Then calculate the inductance LIp, IIs and Im, the accuracy of the calculation about leakage inductance and magnetizing inductance affect the resonance properties directly, so, in this paper, 3D finite element method is used to calculate the leakage inductance and magnetizing inductance. The electromagnetic coupling of two magnetic field distribution is shown in Figure 3. When underwater device is charging in station, after subjected to water, primary and secondary magnetic core will 
produce axial and radial offset with a greater impact on the coupling coefficient, which affects the system power factor and output power. Therefore, the variation trend of the leakage inductance and magnetizing inductance in the disturbance currents is required to research further to optimize the system structure and improve charging efficiency and system stability.

\section{Proposed to optimize the design process}

\subsection{Finite element model established}

The result of electromagnetic coupler leakage inductance and self-inductance in disturbance currents is shown in Table 1.

Table1 plate-type electromagnetic coupling leakage and magnetizing inductance calculation FEM

\begin{tabular}{|c|c|c|c|c|}
\hline $\begin{array}{c}\text { Gap } \\
(\mathrm{m} \\
\mathrm{m})\end{array}$ & $\begin{array}{c}\text { Axial offset } \\
\text { magnetizing } \\
\text { inductance } \\
(\mu \mathrm{H})\end{array}$ & $\begin{array}{c}\text { Axial offset } \\
\text { the leakage } \\
\text { inductance } \\
(\mu \mathrm{H})\end{array}$ & $\begin{array}{c}\text { Magnetizing } \\
\text { inductance } \\
\text { radial } \\
\text { eccentricity } \\
(\mu \mathrm{H})\end{array}$ & $\begin{array}{c}\text { Radial } \\
\text { eccentricity } \\
\text { leakage } \\
\text { inductance } \\
(\mu \mathrm{H})\end{array}$ \\
\hline 0 & 231.1615 & 83.13406 & 231.1615 & 83.13406 \\
\hline 2 & 229.2786 & 84.30426 & 233.126 & 83.04069 \\
\hline 4 & 225.7478 & 86.50307 & 240.0829 & 82.67915 \\
\hline 6 & 221.7337 & 89.90903 & 256.8937 & 82.3921 \\
\hline 8 & 215.7539 & 94.42146 & 295.5942 & 81.74833 \\
\hline 10 & 210.2422 & 100.2297 & 293.4168 & 80.40701 \\
\hline
\end{tabular}

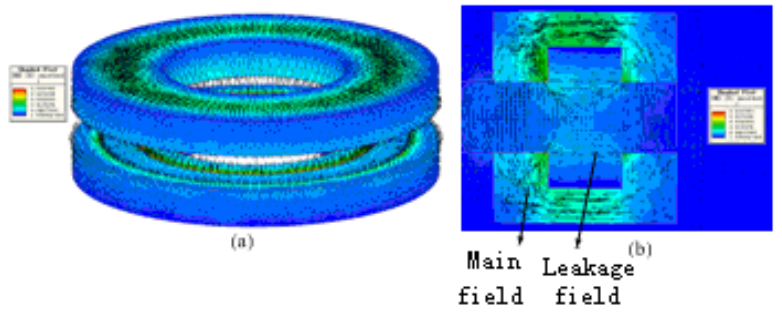

(a) Flat-type coupler 3D electromagnetic field distribution

(b) electromagnetic coupling plate type axial magnetic field distribution section

Figure 3. plate-type electromagnetic field distribution coupler

For resistive $\mathrm{R}_{\text {eq }}$ load, rectifier and filter circuit can be approximated as an equivalent resistance:

$$
R_{\mathrm{e} q}=8 V_{0}^{2} /\left(\pi^{2} P_{L}\right)
$$

To calculate the capacitance $\mathrm{C}_{\mathrm{p}}$ and $\mathrm{C}_{\mathrm{s}}$, the resonance condition depends on the leakage inductance and operating frequency.

$$
\begin{aligned}
& \omega L_{l p}-\frac{1}{\omega C_{p}}=0 \Rightarrow C_{p}=\frac{1}{\omega^{2} L_{l p}} \\
& \omega L_{l s}-\frac{1}{\omega C_{s}}=0 \Rightarrow C_{s}=\frac{1}{\omega^{2} L_{l s}}
\end{aligned}
$$

\subsection{Analysis of the equivalent circuit model}

Each component of the impedance equivalent circuit are as follows:

$$
\begin{aligned}
& Z_{3}=R_{e q}+Z_{s} \\
& Z_{2}=\frac{Z_{m}\left(R_{e q}+Z_{s}\right)}{Z_{m}+R_{e q}+Z_{s}} \\
& Z_{1}=\frac{Z_{m}+R_{e q}+Z_{s}}{Z_{p}\left(Z_{m}+R_{e q}+Z_{s}\right)+\left(R_{e q}+Z_{s}\right) Z_{m}}
\end{aligned}
$$

Among them:

$$
\begin{aligned}
& Z_{p}=R_{p}+j X_{p}=R_{p}+j\left(\omega L_{l p}-\frac{1}{\omega C_{p}}\right) \\
& Z_{s}=R_{s}+j X_{s}=R_{s}+j\left(\omega L_{l s}-\frac{1}{\omega C_{s}}\right) \\
& Z_{m}=j \omega L_{m}
\end{aligned}
$$

\section{3 voltage gain calculation}

Voltage gain is mainly determined by the load, frequency decision. Flowing through the load determines the current of both sides of the electromagnetic coupling. Frequency determines the leakage inductance, magnetizing inductance and impedance value compensation capacitor, and further influence the voltage gain.

$$
G_{V}=\left|\frac{R_{e q}}{Z_{3}} \frac{Z_{2}}{Z_{1}}\right|=\left[(a c-b d)^{2}+(b c+a d)^{2}\right]^{-\frac{1}{2}}
$$

$$
\begin{aligned}
& \frac{R_{e q}}{Z_{3}}=\frac{R_{\mathrm{e} q}}{R_{e q}+Z_{s}} \\
& \frac{Z_{2}}{Z_{1}}=\frac{Z_{m}\left(R_{e q}+Z_{s}\right)}{Z_{p}\left(R_{e q}+Z_{m}+Z_{s}\right)+Z_{m}\left(R_{e q}+Z_{s}\right)}
\end{aligned}
$$

Among them:

$$
\begin{aligned}
& a=1+\frac{R_{s}}{R_{e q}} \\
& b=\frac{X_{s}}{R_{e q}}
\end{aligned}
$$




$$
\begin{gathered}
c=1+\frac{R_{p} R_{m}+X_{p} X_{m}}{R_{m}^{2}+X_{m}^{2}}+\frac{R_{p}\left(R_{e q}+R_{s}\right)+X_{p} X_{s}}{\left(R_{e q}+R_{s}\right)^{2}+X_{s}^{2}} \\
d=\frac{R_{m} X_{p}-R_{p} X_{m}}{R_{m}^{2}+X_{m}^{2}}+\frac{X_{p}\left(R_{e q}+R_{s}\right)-R_{p} X_{m}}{\left(R_{e q}+R_{s}\right)^{2}+X_{s}^{2}}
\end{gathered}
$$

\subsection{Power dissipation and efficiency}

In the electromagnetic coupling, the energy loss includes hysteresis loss, eddy current losses and conduction losses. Hysteresis loss is very small, negligible. As the conductivity of the water is much larger than the core, the eddy current loss is mainly sea eddy current loss. Eddy current loss is proportional to the magnetic field intensity and frequency, so the eddy current loss in the coil is concentrated in the water window. When the coil window, using rubber septa to seal protect, the water eddy current loss can be negligible. Therefore, the electromagnetic coupling of the power loss is mainly conduction losses. Conduction losses include: coil loss, capacitance loss, switching loss, rectifier diode loss and so on.

$$
P_{\text {loss }}=I_{P}^{2}\left(R_{p}+R_{c p}+2 R_{I G B T}\right)+I_{s}^{2}\left(R_{s}+R_{c s}+2 R_{d o n}\right)
$$

The loss can be calculated efficiency:

$$
\eta=\frac{I_{s}^{2} R_{e q}}{P_{l o s s}+I_{s}^{2} R_{e q}}
$$

\section{System Performance Analysis}

To analysis System performance, the parameter values of the electromagnetic coupler designed is given at first. The value in detail is shown in Table 2 .

Table 2 flat electromagnetic coupler design parameters

\begin{tabular}{|c|c|}
\hline Design variables & Parameter values \\
\hline$N_{p}, N_{s}$ & 20 \\
\hline$f(\mathrm{kHz})$ & 20 \\
\hline$C_{p}, C_{s}(\mu F)$ & 0.57 \\
\hline$V_{i}(\mathrm{~V})$ & 70 \\
\hline$V_{o}(\mathrm{~V})$ & 48 \\
\hline$R_{p}, R_{s}$ & 0.029 \\
\hline
\end{tabular}

According to the design parameters, refer to the previous method analysis, the calculation of the flat -type electromagnetic coupling performance in the case of the eccentric is shown in Figure 5, the performance of the offset in the axial direction as shown in Figure 6 , such as load capacity Figure 7 .

In the field of Voltage gain, as can be shown from Figure 5, the flat -type electromagnetic coupling in the eccentric offset has good adaptability, when eccentricity is less than $10 \mathrm{~mm}$, the voltage gain remains stable at $5.8 \%$. As can be seen from Figure 6, the plate -type electromagnetic coupling is sensitive to the axial offset, when the distance is larger or smaller, the error of the voltage gain is larger. But in practical, the difference can be controlled in the lower range by installing the travel switch control between the base station and underwater device. As shown in Figure 7, the electromagnetic coupling can be flat to achieve a stable output voltage over a wide load range. Furthermore, considering the efficiency, in the case of eccentric, the theoretical efficiency of the flat-type electromagnetic coupling is about $90 \%$. However, for axial displacement, there is an optimum position of efficiency.

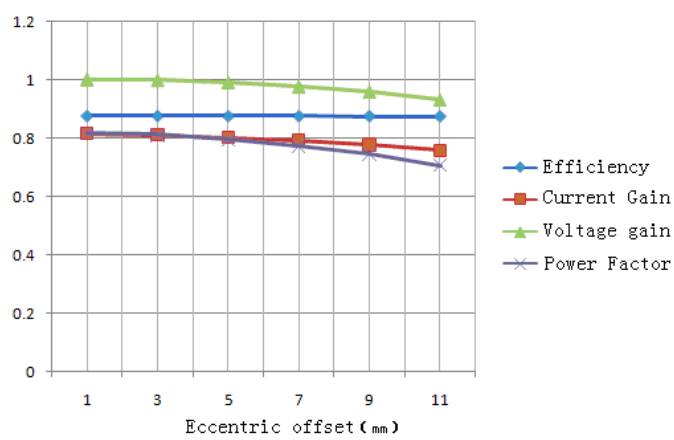

Figure 4 eccentricity offset

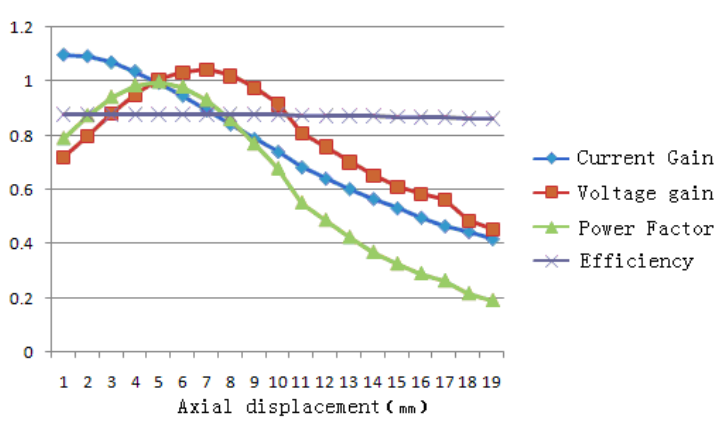

Figure 5 axial offset

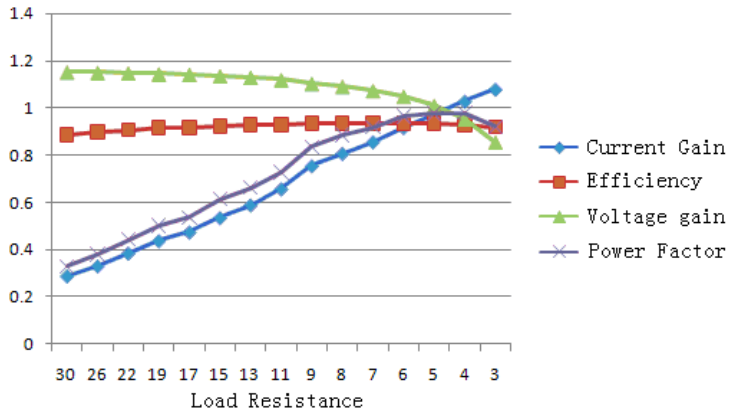

Figure 6 load capacity 


\section{Conclusions}

Application of inductive power transmission technology of underwater equipment for charging can improve the safety and reliability of charge, which can avoid leakage, short circuit and other security risks. This paper presents a novel tablet device suitable for underwater electromagnetic coupling. The electromagnetic coupling can be charged to stable mechanical and electrical equipment underwater currents in the presence of disturbances. Combined with the finite element method and the equivalent circuit, system performance is analyzed and forecasted. The results show that the voltage gain and the charging efficiency of the magnetic coupler is stable, when eccentricity is less than $10 \mathrm{~mm}$, the voltage gain remains stable at $5.8 \%$, and maintained at about $90 \%$ efficiency. The results show that the proposed electromagnetic coupling with high charging efficiency and a stable charging voltage have broad application prospects in charge of underwater equipment .

\section{References}

1. Bradley A M, Feezor M D, Singh H et al. Power systems for autonomous underwater vehicles[J]. IEEE Journal of Oceanic Engineering,26(4),526-538, 2001).

2. Feezor M D, Sorrell F Y, Blankinship P R. An interface system for autonomous undersea vehicles[J]. IEEE Journal of Oceanic Engineering, 26(4),522-525(2001).
3. Kojiya T, Sato F, Matsuki H et al. Construction of non-contacting power feeding system to underwater vehicle utilizing electromagnetic induction $[\mathrm{J}]$. in Oceans 2005 - Europe,1,709-712(.2005).

4. Kojiya T, Sato F, Matsuki H et al. Automatic power supply system to underwater vehicles utilizing non-contacting technology[C]. in Oceans '04 Mts/IEEE Techno-Ocean,04, 2341-2345(2004).

5. McGinnis T, Henze C P, Conroy K. Inductive power system for autonomous underwater vehicles[C]. in 2007 Oceans, Vols 1-5, 736-740(2007).

6. Yoshioka D, Sakamoto H, Ishihara Y et al. Power feeding and data-transmission system using magnetic coupling for an ocean observation mooring buoy[J]. IEEE Transactions on Magnetics, 43(6),2663-2665(2007).

7. Jie Lin, Xing-fei Li et al. Construction of Contactless Power Feeding System for Ocean Buoy[C]. In WiCOM, $20117^{\text {th }}$ International Conference, 1-4(2011).

8. Zhang Yi, Zhou Bing Yin, Hu Guangbo. Downhole linear motor pump fault detector hardware system design $[\mathrm{J}]$. Computer and Digital Engineering, 40(11),162-166(2012).

9. M Behzad, ABastami, R. Maassoumian M. Fault Diagnosis of Centrifugal Pump by Vibration Analysis[C]. Proceedings of 7th Biennial Conference on Engineering System Design an analysis. 3, 221-226(2004). 